\title{
Arquivos deslocados? Em torno dos fundos madeirenses reivindicados ao Arquivo Nacional da Torre do Tombo
}

\author{
L. S. Ascensão de Macedo ${ }^{1}$ \\ Centro de Estudos Interdisciplinares do Século XX, \\ Universidade de Coimbra
}

\section{Introdução}

A restituição de bens culturais às comunidades originárias constitui um tema atual e controverso, que divide sociedades, instituições e o meio académico e/ou profissional. Trata-se de um tema que tem evoluído consideravelmente em diversos domínios, especialmente no âmbito das políticas públicas e do debate académico ${ }^{2}$.

As instituições de memória, principalmente arquivos, bibliotecas e museus, são as entidades sobre as quais recaem com maior incidência este tipo de conflitos de custódia. A custódia constitui um tópico fundamental em domínios aplicados da Ciência da Informação, especialmente no debate entre os paradigmas custodial e pós-custodial na Arquivística $^{3}$. Na terminologia arquivística, o conceito 'custódia' é definido como "guarda física de documentos e/ou arquivos, com a consequente responsabilidade jurídica, sem necessariamente implicar a sua propriedade"4, definição similar a "responsabilidade jurídica, temporária ou definitiva, de guarda e proteção de documentos dos quais não se detém a propriedade", , ou "responsabilidade jurídica de guarda e proteção de arquivos, independentemente de vínculo de propriedade" 6 . O que está em causa consiste em mecanismos de aquisição destes bens culturais, especialmente aqueles ocorridos em contextos específicos no passado, e nas estratégias de (não) representação da informação destes bens. Muitas destas conjunturas ocorreram no passado por motivo

\footnotetext{
${ }^{1}$ Ciência ID: 4D16-AC35-A1DC; ORCID ID: 0000-0001-7251-7314.

${ }^{2}$ Emma Waterton e Steve Watson, "Heritage as a Focus of Research: Past, Present and New Directions", in The Palgrave Handbook of Contemporary Heritage Research, London, Palgrave Macmillan UK, 2015, p. 1-17 (versão eletrónica, consultada a 4 de janeiro de 2019, em https://doi.org/10.1057/9781137293565_1).

${ }^{3}$ Cf. M. C. V. Freitas, “Arquivística Custodial Ou Pós-Custodial? Eis a Questão!”, in Maria José Vicentini Jorente e Dunia Llanes Padrón (eds.), Una Mirada a La Ciencia de La Información Desde Los Nuevos Contextos Paradigmáticos de La Posmodernidad, Marília, Laboratório Editorial UNESP, 2018, p. 13-42; Fernanda Ribeiro, "Archival Science and Changes in the Paradigm", Archival Science, vol. 1, n. ${ }^{\circ}$ 3, 2001, 295-310 (versão eletrónica, consultada a 4 de janeiro de 2019, em https://doi.org/10.1007/BF02437693); Fernanda Ribeiro, "Una Archivística En La Era Post-Custodial: Reflexiones Sobre Un Cambio Del Paradigma En Curso", Boletín Ciencias de La Información Gestión Documental: Directrices Generales Para Procedimientos Archivísticos, vol. 2, 2013, p. 77-82 (versão eletrónica, consultada a 4 de janeiro de 2019, em https://repositorio-aberto.up.pt/handle/10216/77176); Armando Malheiro da Silva e Fernanda Ribeiro, "Documentation/Information and Their Paradigms: Characterization and Importance in Research, Education, and Professional Practice", KO Knowledge Organization, 2012 (versão eletrónica, consultada a 4 de janeiro de 2019, em https://www.nomos-elibrary.de/10.5771/0943-7444-2012-2111/documentation-information-and-their-paradigms-characterization-and-importance-in-researcheducation-and-professional-practice-jahrgang-39-2012-heft-2).

${ }^{4}$ Ivone Alves, Margarida Maria Ortigão Ramos, e Maria Magdalena Garcia, Dicionário de Terminologia Arquivística, Lisboa, Instituto da Biblioteca Nacional e do Livro, Organismo de Normalização Sectorial para a Informação e Documentação, 1993, p. 29.

5 Ana Maria de Almeida Camargo e Heloísa Liberalli Bellotto (eds.), Dicionário de Terminologia Arquivística, São Paulo, Associação dos Arquivistas Brasileiros, Núcleo Regional de São Paulo; Secretaria de Estado da Cultura, 1996, p. 21.

${ }^{6}$ Arquivo Nacional do Brasil, Dicionário Brasileiro de Terminologia Arquivística, Rio de Janeiro, Arquivo Nacional, 2005, p. 62.
} 
de conflitos $\operatorname{armados}^{7}$, do tráfico ilícito ${ }^{8}$, de processos secessionistas ou de descolonização, com consequências na reorganização político-administrativa do território $^{9}$, ou de decisões ope legis emanadas pelo poder central ou, ainda, por determinadas conjunturas políticas, incidindo especialmente sobre comunidades locais ou comunidades indígenas ${ }^{10}$. A dimensão desta disputa pode envolver tanto dois ou mais países como pode ocorrer dentro de uma nação ${ }^{11}$.

No caso dos arquivos, tem havido especial atenção por instituições intergovernamentais ou não-governamentais internacionais e por cada país em matéria de proteção dos seus bens arquivísticos ${ }^{12}$. O Conselho Internacional de Arquivos lançou recomendações específicas nos Princípios básicos sobre o papel dos arquivistas na defesa dos direitos humanos $^{13}$ e reconhece a restituição de arquivos como um princípio básico de justiça e de respeito mútuo.

\footnotetext{
${ }^{7}$ Roger O'Keefe, The Protection of Cultural Property in Armed Conflict, Cambridge, Cambridge University Press, 2006; Jiři Toman, The Protection of Cultural Property in the Event of Armed Conflict: Commentary on the Convention for the Protection of Cultural Property in the Event of Armed Conflict and Its Protocol, Signed on 14 May 1954 in The Hague, and on Other Instruments of Inter, Brookfield, Dartmouth Publishing Company, 1996, (versão eletrónica, consultada a 4 de janeiro de 2019, em https://digitalcommons.law.scu.edu/monographs/11).

${ }^{8}$ Joris Kila e Marc Balcells, "Conclusion: Cultural Property Crime", in Cultural Property Crime: An Overview and Analysis on Contemporary Perspectives and Trends, Leiden, Boston, Brill, 2015, p. 366-70.

${ }^{9}$ Andrzej Jakubowski, State Succession in Cultural Property, Oxford, Oxford University Press, 2015.

${ }^{10}$ Karolina Kuprecht, Indigenous Peoples' Cultural Property Claims, Cham, Springer, 2016.

${ }^{11}$ Joseph P. Fishman, "Locating the International Interest in Intranational Cultural Property Disputes", Yale Journal of International Law, vol. 35, n.. 2, 2010, 347-404; Joe Watkins, "Cultural Nationalists, Internationalists, and "Intra-Nationalists"": Who's Right and Whose Right?"', International Journal of Cultural Property, vol. 12, n. ${ }^{\circ}$ 1, 2009, 78-94 (versão eletrónica, consultada a 4 de janeiro de 2019, em https://doi.org/doi.org/10.1017/S0940739105999992).

${ }^{12}$ Há um corpus jurídico internacional que contempla a proteção e restituição dos arquivos, como a Convenção de Haia de 1954, a Convenção da UNESCO de 1970, a UNIDROIT, as Resoluções do Parlamento Europeu e Convenção de Viena. É de realçar, também, o papel do Conselho Internacional de Arquivos que tem desenvolvido ações determinantes para a identificação de casos de contencioso arquivístico e de recomendações procedentes do Grupo de Peritos para os Assuntos Jurídicos e do recente Grupo de Peritos para o Património Arquivístico Partilhado. Cf. UNESCO, Convention for the Protection of Cultural Property in the Event of Armed Conflict with Regulations for the Execution of the Convention, The Hague, 14 May 1954, 1954; UNESCO, Convention on the Means of Prohibiting and Preventing the Illicit Import, Export and Transfer of Ownership of Cultural Property Adopted by the General Conference at Its Sixteenth Session, Paris, 14 November 1970, Paris, UNESCO, 1970; UNIDROIT, UNIDROIT convention on stolen or illegally exported cultural objects, Rome, 24 June 1995, 1995; Parlamento Europeu, Resolução sobre o direito dos povos à informação sobre a sua História e à restituição dos arquivos nacionais (Jornal Oficial das Comunidades Europeias, 1991), p. 181-206; Parlamento Europeu, Diretiva 2014/60/UE do Parlamento Europeu e do Conselho, de 15 de maio de 2014, relativa à restituição de bens culturais que tenham saído ilicitamente do território de um Estado-Membro e que altera o Regulamento (UE) n. ${ }^{\circ}$ 1024/2012 (Reformulação), Jornal Oficial da União Europeia, 2014 (versão eletrónica, consultada a 4 de janeiro de 2019, em https://eur-lex.europa.eu/legalcontent/pt/TXT/?uri=CELEX\%3A32014L0060); United Nations, Vienna Convention on Succession of States in respect of State Property, Archives and Debts, 1983-04-08, United Nations, 2005; Leopold Auer, Disputed Archival Claims: Analysis of an International Survey: A RAMP Study, UNESCO, Paris, UNESCO, 1998; International Council on Archives, "The View of the Archival Community on the Settling of Disputed Claims: Position Paper Adopted by the Executive Committee of the International Council on Archives, Guangzhou, China, 10-13 April 1995”, ICA/CIA, 1995; International Council on Archives, "Reference Dossier on Archival Claims", in Hervé Bastien (ed.), Proceedings of the twenty-nineth, thirtieh and thirty first International Conference of the Round Table on Archives. Special Issue JANUS. Dordrecht 1998, 2005, p. 209-268.

${ }^{13}$ International Council on Archives, Basic Principles on the Role of Archivists and Records Managers in Support of Human Rights, International Council on Archives, 2016 (versão eletrónica, consultada a 4 de janeiro de 2019, em https://www.ica.org/en/basic-principles-role-archivists-and-records-managerssupport-human-rights).
} 
No entanto, a produção científica sobre arquivos deslocados é esparsa. Para Macedo ${ }^{14}$, uma das principais dificuldades em torno da condição destes arquivos é de natureza conceptual. A Society of American Archivists define "arquivos deslocados" (displaced archives) como "documentos de valor permanente que foram removidos do controlo do seu proprietário legítimo" 15 . A nota desta entrada identifica termos sinónimos, como alienated record, expatriate archives, fugitive record, migrated archives. A pesquisa preliminar feita na Multilingual Archival Terminology, repositório eletrónico que congrega a terminografia arquivística internacional disponível em diversas línguas, não apresenta qualquer entrada referente a displaced archives, apresentando outros, como fugitive archives, captured archives ou removed archives ${ }^{16}$. Curiosamente, a terminografia arquivística de língua portuguesa disponível não disponibiliza qualquer entrada sobre 'arquivos deslocados' ${ }^{17}$. As únicas exceções podem ser encontradas em 'arquivo deslocado' em Camargo e Bellotto ${ }^{18} \mathrm{e}$ 'arquivo removido' em $\mathrm{Nagel}^{19}$, que se baseiam na tradução de textos terminográficos do Conselho Internacional de Arquivos. A terminografia arquivística portuguesa disponibiliza somente 'contencioso arquivístico' (disputed archival claims ou replevin), definido como "litígio sobre jurisdição arquivística e/ou direito de propriedade de arquivos" ${ }^{20}$. Esta diversidade conceptual ocorre também noutras línguas. De acordo com Karabinos, esta multiplicidade de denominações derivou do facto de os arquivos deslocados terem sido abordados academicamente por "non-archivists in non-archival terms" "21, especialmente por historiadores, juristas e jornalistas. Porém, a perceção da comunidade arquivística sobre os denominados 'arquivos deslocados' não está isenta de equívocos. Ketelaar referiu que "[a]rchives are always displaced, that is (in day-to-day language), removed from place A to place B" (2017, p. viii). Esta afirmação sugere que os arquivos, como categoria de bens móveis, são passíveis de serem transferidos para espaços distintos do local de criação e/ou de acumulação. No entanto, os arquivos deslocados permitem associar, por via do senso comum, à ideia de documentos ou conjuntos documentais que se encontram no lugar errado. Esta e outras variantes, inspiradas na metáfora dos movimentos migratórios, não clarificam a dimensão do problema. Definir o estatuto destes bens arquivísticos que foram desarraigados do seu habitat natural, total ou parcialmente, é essencial para explorar a dimensão do conflito. O que está em causa consiste em fenómenos de despossessão de bens culturais às comunidades que reivindicam pelo seu direito à memória, à continuidade

\footnotetext{
${ }^{14}$ L. S. Ascensão de Macedo, "Repatriação Dos Arquivos Ou Reunificação Virtual? O Caso Dos Fundos Conventuais Madeirenses Dispersos Entre o Arquivo Nacional Torre Do Tombo e o Arquivo Regional e Biblioteca Pública Da Madeira", in Maria Manuel Borges e Elias Sanz Casado (eds.), VIII Encontro Ibérico EDICIC 2017, Coimbra 2017-11-20/2017-11-22, Coimbra: Universidade de Coimbra, 2017, p. 1325-1344. 15 Dictionary Working Group of the Society of American Archivists, "Displaced Archives", Word of the Week: Dictionary of Archives Terminology, 2018 (versão eletrónica, consultada a 4 de janeiro de 2019, em https://us3.campaign-archive.com/?u=56c4cfbec1ee5b2a284e7e9d6\&id=1993e03415).

${ }^{16}$ International Council on Archives, "DAT III: Dictionary of Archival Terminology", 2004; International Council on Archives, "Multilingual Archival Terminology", 2013 (versão eletrónica, consultada a 4 de janeiro de 2019, em http://www.ciscra.org/mat/mat).

17 Biblioteca Nacional de Portugal, Normas Portuguesas de Documentação e Informação CT 7, Lisboa, BNP, IPQ, 2010.

${ }^{18}$ Camargo e Bellotto, Op. cit.

${ }^{19}$ Rolf Nagel (ed.), Dicionário de Termos Arquivísticos: Subsídios Para Uma Terminologia Arquivística Brasileira, ed. by Rolf Nagel, Bonn, Salvador, Deutsche Stiftung für internationale Entwicklung: Universidade Federal da Bahia, 1989.

${ }^{20}$ Alves, Ramos, e Garcia, Op. cit., p. 24.

${ }^{21}$ Michael Karabinos, "Archives and Post-Colonial State-Sponsored History: A Dual State Approach Using the Case of the "Migrated Archives"', in The Palgrave Handbook of State-Sponsored History After 1945, London, Palgrave Macmillan UK, 2018, p. 177-90 (versão eletrónica, consultada a 4 de janeiro de 2019, em https://doi.org/10.1057/978-1-349-95306-6_9).
} 
histórica, ao acesso à informação e ao património cultural ${ }^{22}$. Perfillhamos a definição de 'arquivos deslocados' do Grupo de Trabalho para os Arquivos e Direitos Humanos do Conselho Internacional de Arquivos, que entende como "arquivos que foram transferidos e estão sob a custódia de pessoa ou instituição que, legalmente, não tem direito a eles" 23 . Esta comunicação versa-se sobre um caso concreto referente aos pedidos de restituição de arquivos reivindicados pela Região Autónoma da Madeira (RAM) ${ }^{24}$ ao Arquivo Nacional da Torre do Tombo (ANTT). Reconhecendo que os casos de restituição de arquivos em contexto intranacional constituem uma dimensão pouco abordada na produção científica em Portugal, a nossa análise incide especialmente sobre as estratégias de identificação e de representação da informação dos conjuntos documentais reivindicados pela RAM. Como tal, pretende-se responder a duas perguntas de investigação:

(1) Quais são os fundos reivindicados pelas autoridades madeirenses ao ANTT?

(2) Como é que estes conjuntos documentais se encontram representados nos instrumentos de acesso à informação do ANTT e da RAM, especialmente no que respeita à FIA?

Não podemos falar de "Lugares Pioneiros" sem reconhecer o papel insubstituível dos arquivos na construção da memória das comunidades. Como este estudo não tem como ânimo resolver o problema da restituição dos arquivos em disputa, dado que consiste um problema do domínio político e institucional, o objetivo deste estudo consiste em identificar os factores incoativos da despossessão dos arquivos madeirenses custodiados pelo ANTT e pela RAM a partir dos IAI.

\section{Breve contextualização do problema}

Na sequência das comemorações dos 600 anos do descobrimento e do povoamento das ilhas do Porto Santo e da Madeira, a Resolução da Assembleia Legislativa da Madeira n. ${ }^{\circ}$ $3 / 2017 / \mathrm{M}$, de 12 de janeiro, recomendou ao Governo da República para que tome as diligências necessárias para "proceder à transferência dos documentos produzidos por instituições regionais que se encontram na Torre do Tombo para o Arquivo Regional da Madeira" 25 . Trata-se de um pedido formal feito por um organismo legislativo da administração regional autónoma para a restituição de arquivos que foram transferidos no último quartel do século XIX para o Arquivo Nacional da Torre do Tombo (ANTT). Por outras palavras, estamos na presença de um pedido de restituição de arquivos em contexto intranacional.

Desde a criação do Arquivo Distrital do Funchal (ADF), através do Decreto n. ${ }^{\circ} 19952$ de 30 de julho de 1931, que retificou o Decreto n. ${ }^{\circ}$ 19952, de 27 de junho de $1931^{26}$, foi João

\footnotetext{
${ }^{22}$ Brigitta Hauser-Schäublin e Lyndel V. Prott, "Changing Concepts of Ownership, Culture and Property: Introduction", in Brigitta Hauser-Schäublin e Lyndel V. Prott (eds.), Cultural Property and Contested Ownership: The Trafficking of Artefacts and the Quest for Restitution, New York, Routledge, 2016, p. 120.

${ }^{23}$ International Council on Archives, Basic Principles on the Role of Archivists and Records Managers in Support of Human Rights, p. 16.

${ }^{24}$ Todas as vezes que nos referirmos à sigla RAM faremos correspondência com as entidades Assembleia Legislativa da Região Autónoma da Madeira e Governo Regional da Madeira.

25 Assembleia Legislativa da Região Autónoma da Madeira, Resolução n. ${ }^{\circ}$ 3/2017/M, de 12 de Janeiro, Portugal, Jornal Oficial da Região Autónoma da Madeira, 2017, p. 409 (versão eletrónica, consultada a 4 de janeiro de 2019, em https://data.dre.pt/eli/resolalram/3/2017/01/12/m/dre $/ \mathrm{pt} / \mathrm{html}$ ).

${ }^{26}$ Portugal. Leis Decretos, "Decreto n. ${ }^{\circ}$ 19952, de 27 de Junho", Diário do Govêrno, n. ${ }^{\circ}$ 147/1931, Série I de 1931-06-27.
} 
Cabral do Nascimento, seu primeiro diretor, que efetuou formalmente, em 1932, o primeiro pedido de restituição dos fundos custodiados pelo ANTT à Inspeção Geral das Bibliotecas e Arquivos (IGBA). Em janeiro de 1933, a IGBA declinou a possibilidade de restituição. Em 1937, o ADF descobriu os mesmos conjuntos documentais remanescentes que foram transferidos para o ANTT. Esta situação suscitou o problema de reunificação dos fundos, com consequências não só em termos de acesso à informação como também de acesso ao património documental pela comunidade insular. Este problema ainda persiste até aos dias de hoje, sendo o Arquivo Regional e Biblioteca Pública da Madeira $(\mathrm{ABM})$ a entidade sucessora do ADF.

\section{Materiais e métodos}

Para responder às questões de investigação supramencionadas, propomos uma abordagem mista com enfoque predominantemente qualitativo ${ }^{27}$. Esta estratégia possibilita combinar dados sobre a representação do conteúdo de conjuntos documentais em IAI publicados pelo ANTT e pelo ABM (dados qualitativos) com o grau de dispersão das unidades documentais (dados quantitativos).

Para a identificação dos arquivos reivindicados pela Resolução da Assembleia Legislativa da Madeira n. ${ }^{\circ}$ 3/2017/M, de 12 de janeiro, a análise de IAI possibilita-nos explorar estratégias de representação dos conjuntos documentais em disputa. Para Ribeiro, a representação da informação arquivística materializada em IAI (genologicamente materializados em catálogos, inventários, guias, índices ou bases de dados) continua a ser aplicada pela comunidade profissional para servir uma dupla função, por um lado, para "organizar/arrumar (fisicamente) documentos" e, por outro, para "representar/recuperar informação" ${ }^{28}$. Neste caso específico, para a questão (1) a nossa análise incide na extração de dados referentes à zona de identificação (metadados Código(s) de referência, Título, Data(s), Nivel de descrição, Dimensão e suporte) da norma internacional $\operatorname{ISAD}(\mathrm{G})^{29}$. Do ponto de vista do nível de descrição, cingir-nos-emos ao nível de fundo ou de sub-fundo. Para a contextualização dos processos de aquisição, interessa-nos particularmente o metadado fonte imediata de aquisição ou transferência (FIA) da zona de contexto para responder à questão (2). Convém reconhecer que os IAI têm evoluído consideravelmente, tanto em suporte analógico como em suporte digital ${ }^{30}$. A pesquisa decorreu através de uma recolha em bases de dados ou listagens disponíveis no portal institucional Portal

\footnotetext{
${ }^{27}$ Sharlene Nagy Hesse-Biber, Deborah Rodriguez, e Nollaig Frost, “A Qualitatively Driven Approach to Multimethod and Mixed Methods Research", in Sharlene Nagy Hesse-Biber e R. Burke Johnson (eds.), The Oxford Handbook of Multimethod and Mixed Methods Research Inquiry, Oxford, Oxford University Press, 2015, p. 3-20 (versão eletrónica, consultada a 4 de janeiro de 2019, em https://doi.org/10.1093/oxfordhb/9780199933624.001.0001).

28 Fernanda Ribeiro, "O Uso Da Classificação Nos Arquivos Como Instrumento de Organização, Representação e Recuperação Da Informação”, 2013 (versão eletrónica, consultada a 4 de janeiro de 2019, em https://repositorio-aberto.up.pt/handle/10216/69659).

${ }^{29}$ Conselho Internacional de Arquivos, $\operatorname{ISAD}(G)$ : Norma Geral Internacional de Descrição Arquivística : Adoptada Pelo Comité de Normas de Descrição, Estocolmo, Suécia, 19-22 de Setembro de 1999., 2.", Lisboa: Instituto dos Arquivos Nacionais/Torre do Tombo, 2002.

${ }^{30}$ Richard J. Cox, "Revisiting the Archival Finding Aid", Journal of Archival Organization, vol. 5, n. ${ }^{\circ}$ 4, 2008, p. 5-32 (versão eletrónica, consultada a 4 de janeiro de 2019, em https://doi.org/10.1080/15332740802153245); Ciaran B. Trace e Andrew Dillon, "The Evolution of the Finding Aid in the United States: From Physical to Digital Document Genre", Archival Science, vol. 12,

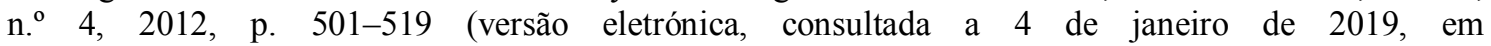
https://doi.org/10.1007/s10502-012-9190-5); H MacNeil, "What Finding Aids Do: Archival Description as Rhetorical Genre in Traditional and Web-Based Environments", Archival Science, vol. 12, n. ${ }^{\circ}$ 4, 2012 , p. 485-500 (versão eletrónica, consultada a 4 de janeiro de 2019, em https://doi.org/10.1007/s10502-0129175-4).
} 
Português de Arquivos (PPA) ${ }^{31}$ e no $\mathrm{ABM}^{32}$. Nos motores de pesquisa do portal institucional web do PPA utilizamos descritores para recuperar informação em título de fundo, utilizando a denominação dos fundos enumerados na Resolução supra exarada ${ }^{33}$, e combinados com "Madeira" e "Funchal". Os resultados foram extraídos e processados em .xlsx (Microsoft). Para suporte à análise de conteúdo, baseámo-nos num conjunto de garantias literárias emanadas pelo Conselho Internacional de Arquivos ${ }^{34}$ e pelo órgão nacional para a gestão dos arquivos ${ }^{35}$, atendendo às recomendações para a descrição do metadado FIA.

\section{Resultados}

\section{Quais são os fundos reivindicados pelas autoridades madeirenses ao ANTT?}

A Resolução n. ${ }^{\circ}$ 3/2017/M, de 12 de janeiro, enumerou cinco fundos a "transferir" para a Região Autónoma da Madeira: "Cabido da Sé do Funchal, do Convento de Santa Clara, do Convento da Encarnação, da Provedoria da Real Fazenda e da Alfândega do Funchal" ${ }^{36}$. Contudo, os resultados da pesquisa efetuada no portal institucional devolveram onze fundos madeirenses na custódia do ANTT, a saber: (1) Convento de Santa Clara do Funchal (CSCF); (2) Convento de Nossa Senhora da Encarnação do Funchal (CNSEF); (3) Convento de São Francisco do Funchal (CSFF); (4) Cabido da Sé do Funchal (CSF); (5) Convento de Nossa Senhora da Piedade de Santa Cruz (CNSPSC); (6) Convento de São Bernardino de Câmara de Lobos (CSBCL); (7) Convento de Nossa Senhora da Porciúncula da Ribeira Brava (CNSPRB); (8) Convento de São Sebastião da Calheta (CSSC); (9) Provedoria e Junta da Real Fazenda do Funchal (PJRFF); (10) Comissão da Fazenda do Distrito da Madeira e Porto Santo (CFMPS) e (11) Alfândega do Funchal (ALF). A tabela I apresenta sinteticamente os dados extraídos do Portal Português de Arquivos.

\footnotetext{
31 Direção-Geral do Livro dos Arquivos e das Bibliotecas, "Portal Português de Arquivos", 2017 (versão eletrónica, consultada a 4 de janeiro de 2019, em https://portal.arquivos.pt/).

${ }^{32}$ Arquivo Regional e Biblioteca Pública da Madeira, Instrumentos Descritivos, Funchal, ABM, 2017.

33 Os descritores utilizados foram: "Cabido da Sé do Funchal", "Convento de Santa Clara", "Convento da Encarnação", "Provedoria da Real Fazenda" e "Alfândega do Funchal".

${ }^{34}$ International Council on Archives, ISAD $(G)$ : General International Standard Archival Description : Adopted by the Committee on Descriptive Standards, Stockholm, Sweden, 19-22 September 1999, Ottawa, International Council of Archives, 2000.

${ }^{35}$ Direcção-Geral de Arquivos, Orientações Para a Descrição Arquivística, $2 .{ }^{a}$ versão, Lisboa, Direcção Geral de Arquivos, Grupo de Trabalho de Normalização da Descrição em Arquivo, 2007.

${ }^{36}$ Assembleia Legislativa da Região Autónoma da Madeira, Op. cit., p. 409.
} 


\begin{tabular}{|c|c|c|c|c|}
\hline Fundo & Datas extremas & $\begin{array}{l}\text { Dimensão } \\
\text { (em u.i., } n=2178 \text { ) }\end{array}$ & $\begin{array}{l}\text { U.i. com } \\
\text { representação digital }\end{array}$ & Fonte \\
\hline 1. ALF & $1620-1834$ & 475 & 462 & 37 \\
\hline 2. $\quad$ CFMPS & $1834-1851$ & 16 & 16 & 38 \\
\hline 3. CNSPRB & 1736-1809 & 3 & 3 & 39 \\
\hline 4. $\mathrm{CNSPSC}$ & $1772-1776$ & 1 & 1 & 40 \\
\hline 5. $\mathrm{CSBCL}$ & $1792-1825$ & 4 & 4 & 41 \\
\hline 6. $\mathrm{CSCF}$ & $1447-[1900]$ & 144 & 114 & 42 \\
\hline 7. $\mathrm{CSFF}$ & 1732-1832 & 7 & 7 & 43 \\
\hline 8. $\quad \mathrm{CSSC}$ & $1674-1811$ & 4 & 3 & 44 \\
\hline 9. $\quad$ PJRFF & $1569-1834$ & 1398 & 1341 & 45 \\
\hline 10. CSF & $1478-1861$ & 78 & 41 & 46 \\
\hline 11. CNSEF & $1660-1890$ & 48 & 48 & 47 \\
\hline
\end{tabular}

Tabela 1 Listagem dos fundos custodiados pelo ANTT. Fonte: Portal Português de Arquivos.

Importa realçar que o Portal Português de Arquivos devolveu os subfundos Confraria das Escravas de Nossa Senhora do Monte (CSCF) e a Confraria de Nossa Senhora Mãe dos Homens e do Patriarca São José (CSFF). Além disto, a base de dados devolveu igualmente os subfundos Subdelegação do Funchal da Secção Internacional da Polícia de Vigilância e Defesa do Estado (PIDE/DGS) e a Comissão Distrital do Funchal da União Nacional, transferidos para Lisboa em 1974 para a custódia do Serviço de Coordenação da Extinção da PIDE/DGS. Estes fundos não serão objeto de análise, por não constarem no teor da Resolução. $O$ facto de a Resolução apenas enumerar cinco fundos só pode ter justificação no recurso a um IAI desatualizado. Desde 2002, o ANTT

37 Arquivo Nacional da Torre do Tombo, "Alfândega Do Funchal”, DIGITARQ, Direcção-Geral de Arquivos, 2008 (versão eletrónica, consultada a 4 de janeiro de 2019, em https://digitarq.arquivos.pt/details?id=3886618).

38 Arquivo Nacional da Torre do Tombo, "Comissão Da Fazenda Do Distrito Da Madeira e Porto Santo", DIGITARQ, Direcção-Geral de Arquivos, 2008 (versão eletrónica, consultada a 4 de janeiro de 2019, em https://digitarq.arquivos.pt/details?id=3909719).

39 Arquivo Nacional da Torre do Tombo, "Convento de Nossa Senhora Da Porciúncula Da Ribeira Brava", DIGITARQ, Direcção-Geral de Arquivos, 2011 (versão eletrónica, consultada a 4 de janeiro de 2019, em https://digitarq.arquivos.pt/details?id=1375741).

40 Arquivo Nacional da Torre do Tombo, "Convento de Nossa Senhora Da Piedade de Santa Cruz: Fundo", DIGITARQ, Direcção-Geral de Arquivos, 2011 (versão eletrónica, consultada a 4 de janeiro de 2019, em https://digitarq.arquivos.pt/details?id=1375743).

41 Arquivo Nacional da Torre do Tombo, "Convento de São Bernardino de Câmara de Lobos: Fundo", DIGITARQ, Direcção-Geral de Arquivos, 2011 (versão eletrónica, consultada a 4 de janeiro de 2019, em https://digitarq.arquivos.pt/details?id=1379034).

42 Arquivo Nacional da Torre do Tombo, "Convento de Santa Clara Do Funchal”, DIGITARQ, DirecçãoGeral de Arquivos, 2011 (versão eletrónica, consultada a 4 de janeiro de 2019, em https://digitarq.arquivos.pt/details?id=1379435).

43 Arquivo Nacional da Torre do Tombo, "Convento de São Francisco Do Funchal: Fundo", DIGITARQ, Direcção-Geral de Arquivos, 2011 (versão eletrónica, consultada a 4 de janeiro de 2019, em https://digitarq.arquivos.pt/details?id=1379962).

44 Arquivo Nacional da Torre do Tombo, "Convento de São Sebastião Da Calheta”, DIGITARQ, DirecçãoGeral de Arquivos, 2011 (versão eletrónica, consultada a 4 de janeiro de 2019, em https://digitarq.arquivos.pt/details?id=1382433).

${ }^{45}$ Arquivo Nacional da Torre do Tombo, "Provedoria e Junta Da Real Fazenda Do Funchal", DIGITARQ, Direcção-Geral de Arquivos, 2008 (versão eletrónica, consultada a 4 de janeiro de 2019, em https://digitarq.arquivos.pt/details?id=4310201).

46 Arquivo Nacional da Torre do Tombo, "Cabido Da Sé Do Funchal”, DIGITARQ, Direcção-Geral de Arquivos, 2011 (versão eletrónica, consultada a 4 de janeiro de 2019, em https://digitarq.arquivos.pt/details?id=1379940).

47 Arquivo Nacional da Torre do Tombo, "Convento de Nossa Senhora Da Encarnação Do Funchal", DIGITARQ, Direcção-Geral de Arquivos, 2011 (versão eletrónica, consultada a 4 de janeiro de 2019, em https://digitarq.arquivos.pt/details?id=1375671). 
empreendeu uma reorganização dos fundos conventuais cujo inventário foi coordenação da arquivista madeirense Maria do Carmo Jasmins Dias Farinha, sob a direção José Mattoso $^{48}$. Basicamente, foram individualizados seis novos fundos que permaneceram indistintos no fundo da PJRFF desde a sua transferência para Lisboa em finais do século XIX, mais concretamente os conventos masculinos (CFMPS, CNSPRB, CNSPSC, CSBCL, CSFF e CSSC) ${ }^{49}$. Sobre os fundos madeirenses identificados na tabela I, importa referir que, desde 1937, o ADF procurou inventariar no ANTT a documentação reivindicada, cujos resultados foram publicados no boletim Arquivo Histórico da Madeira ${ }^{50}$. Os fundos ALF, PJRFF, CNSEF e CSCF foram microfilmados e parcialmente digitalizados a partir do microfilme, projeto conduzido pelo Centro de Estudos de História do Atlântico ${ }^{51}$.

$\mathrm{O}$ portal institucional do $\mathrm{ABM}{ }^{52}$, por seu turno, apenas disponibiliza um inventário digitalizado e não dispõe de qualquer representação digital sobre os conjuntos documentais que custodiam nas bases de dados institucionais. A tabela seguinte enumera os conjuntos documentais constantes desta entidade:

\begin{tabular}{|c|c|c|c|}
\hline Fundo & Datas extremas & $\begin{array}{l}\text { Dimensão } \\
(\text { em u.i.; } n=10680)\end{array}$ & Fonte \\
\hline 1. ALF & $1650-2000$ & 10598 & 53 \\
\hline 2. CNSEF & $1645-1895$ & 36 & 54 \\
\hline 3. CSBCL & $1783-1832$ & 1 & \\
\hline 4. $\mathrm{CSCF}$ & 1634-1897 & 17 & \\
\hline 5. $\mathrm{CSFF}$ & $1710-1730$ & 1 & \\
\hline 6. CNSPSC & $1705-1710$ & 1 & \\
\hline 7. PJRFF & $1649-1833$ & 26 & \\
\hline
\end{tabular}

Tabela 2. Fundos custodiados pelo $\mathrm{ABM}^{55}$.

Conforme se pode verificar através da Tabela II, os conjuntos documentais foram incorporados no ADF (entidade da administração central desconcentrada préautonómica) desde 1937 (CSFF, CNSEF, CSBCL, CSCF, CNSPSC). Sobre a PJRFF, não

\footnotetext{
${ }^{48}$ Arquivo Nacional da Torre do Tombo, Ordens Monástico-Conventuais: Inventário: Ordem de São Bento, Ordem Do Carmo, Ordem Dos Carmelitas Descalços, Ordem Dos Frades Menores, Ordem Da Conceição de Maria, Lisboa, ANTT, 2002.

49 Arquivo Nacional da Torre do Tombo, "Alfândega Do Funchal", in Maria do Carmo Jasmins Dias Farinha e António Frazão, Guia Geral Dos Fundos Da Torre Do Tombo: Instituições Do Antigo Regime, Administração Periférica. Domínios. Casa Real e Anexas, Lisboa, IAN/TT, 2002, p. 356-370.

${ }^{50}$ S.N., "A Madeira Nos Arquivos Nacionais: Alfândega Do Funchal. Parte 1 de 8", Arquivo Histórico Da Madeira, vol. 6, 1939, p. 129-132; S.N., "A Madeira Nos Arquivos Nacionais: Cabido Da Sé Catedral. Parte 2 de 8", Arquivo Histórico Da Madeira, vol. 6, 1939, p. 129-132; S.N., "A Madeira Nos Arquivos Nacionais: Livro Da Repartição Da Fazenda. Parte 6 de 8", Arquivo Histórico Da Madeira, vol. 6, 1939, 160-168; S.N., “A Madeira Nos Arquivos Nacionais: Convento de Santa Clara. Parte 4 de 8”, Arquivo Histórico Da Madeira, vol. 6, 1939, p. 129-132; S.N., "A Madeira Nos Arquivos Nacionais: Convento Da Encarnação. Parte 3 de 8", Arquivo Histórico Da Madeira, vol. 6, 1939, p. 129-132; João Franco Machado, “Alguns Documentos Do Mosteiro de Santa Clara Do Funchal”, Arquivo Histórico Da Madeira, vol. 4, 1935, p. 171-172.

${ }^{51}$ CEHA, "NESOS: Base de Dados de História Das Ilhas Atlânticas", Centro de Estudos de História Do Atlântico, 1995.

52 Governo Regional da Madeira, “ABM - Arquivo Regional e Biblioteca Pública Da Madeira [Portal Institucional]'.

53 ABM, Alfândega Do Funchal: Inventário: Instrumentos Descritivos IDD 3, Funchal, 1990; ABM, Alfândega Do Funchal: Inventário (Cotas 1793 a 10598), Funchal, 2012.

${ }^{54}$ ARM, Inventário Dos Arquivos Dos Conventos, Funchal, Arquivo Regional e Biblioteca Pública da Madeira; ARM, Guia do Arquivo Regional da Madeira, Funchal, Secretaria Regional do Turismos e Cultura, Direcção Regional dos Assuntos Culturais, Arquivo Regional da Madeira, 1997.

${ }^{55}$ Id., ibid.
} 
foi documentado qualquer auto de incorporação anterior a 1957 e, no que respeita ao fundo ALF, a documentação foi incorporada ao longo dos anos em 1951, 1953, 1958, 1964, 1975 e, em contexto autonómico através do ABM, em 2010 e 2012. Apesar da amplitude cronológica, alguns de vários séculos, houve perdas documentais significativas ocorridas no passado devido a factores humanos e naturais, que se pode depreender através de lapsos cronológicos. Os IAI disponibilizados pelo ABM constituem simplesmente listagens de unidades de instalação, que não cumprem na sua totalidade com as normas internacionais de descrição arquivística, sem representações digitais destas unidades nas bases de dados desta entidade ${ }^{56}$. Assim, podemos concluir, através desta breve análise, que o problema presente é o da reunificação de fundos, como o gráfico seguinte elucida.

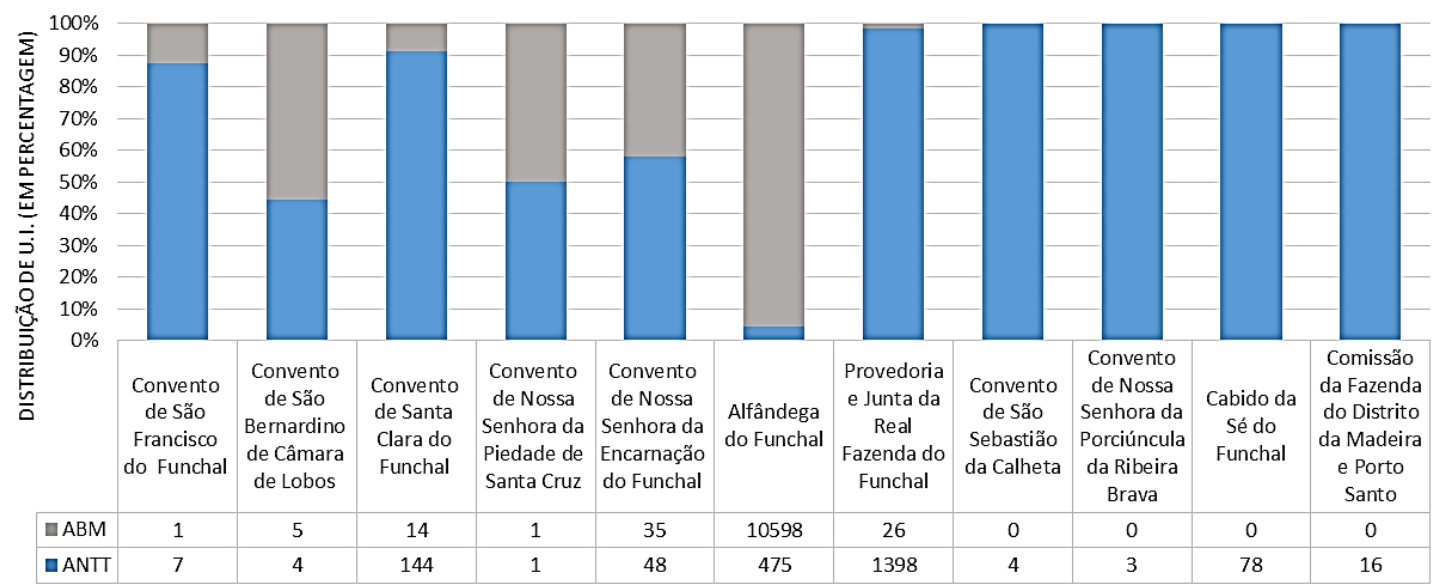

Gráfico 1. Volume (\%) de u.i. com custódia distribuída entre o ABM e o ANTT

Como se pode observar através do Gráfico 1, é visível a presença de dois blocos distintos de conjuntos documentais dispersos (doravante, CDD), a saber:

(1) CDD1: sete fundos repartidos em dupla custódia entre o ABM e ANTT: ALF (ABM $95,7 \%=10598$ u.i. versus ANTT 4, 3\% $=475$ u.i.); $C S B C L(\mathrm{ABM} 55,6 \%=5$ u.i. versus ANTT 44,4\% = 4 u.i.); CNSPSC (ABM 50,0\% $=1$ u.i. versus ANTT 50\% $=1$ u.i.); $C N S E F$ (ABM $42,2 \%=35$ u.i. versus ANTT 57,8\% = 48 u.i.); CSCF (ABM $8,9 \%=14$ u.i. versus ANTT 91, $1 \%=144$ u.i.); $C S F F(\mathrm{ABM} 12,5 \%=1$ u.i. versus ANTT $87,5 \%=7$ u.i.) e PJRFF (ABM 1,8\% $=26$ u.i. versus ANTT 98,2\% $=1398$ u.i.).

(2) CDD2: quatro fundos que se encontram na totalidade numa única entidade custodiante (ANTT): Cabido da Sé do Funchal (78 u.i.), Comissão da Fazenda do Distrito da Madeira e Porto Santo (16 u.i.), Convento de Nossa Senhora da Porciúncula da Ribeira Brava (3 u.i.), Convento de São Sebastião da Calheta (3 u.i.).

Em síntese, a Resolução n. ${ }^{\circ}$ 3/2017/M, de 12 de janeiro, apresenta um número inferior de de fundos reivindicados ao ANTT. Apesar de o ANTT ter realizado uma digitalização disponível no Portal Português de Arquivos sobre os conjuntos documentais reivindicados, o $\mathrm{ABM}$, por seu turno, não possui um inventário atualizado nem os fundos se encontram digitalizados e acessíveis em sistemas de informação de arquivo. O problema subjacente na representação destes arquivos é o da sua reunificação (física e/ou

56 Arquivo Regional e Biblioteca Pública da Madeira, Instrumentos Descritivos, Funchal, 2017; Arquivo Regional e Biblioteca Pública da Madeira, "Arquivo Regional e Biblioteca Pública Da Madeira”, Página Institucional, 2017 (versão eletrónica, consultada a 4 de janeiro de 2019, em https://abm.madeira.gov.pt). 
digital), uma vez que as entidades custodiantes produtoras dos IAI não tiveram preocupações em representar de forma reunificada os conjuntos documentais dispersos.

\section{Como é que estes conjuntos documentais se encontram representados nos instrumentos de acesso à informação do ANTT e da RAM, especialmente no que respeita à FIA?}

O metadado FIA tem como objetivo "identificar a fonte imediata de aquisição ou transferência da unidade de descrição" "57. Como tal, este metadado permite-nos contextualizar nos IAI compulsados os processos referentes às "formas de aquisição, ou seja, de obtenção da propriedade e/ou custódia de documentos de arquivo" 58 , de distintas modalidades como doações, depósitos, heranças, legados, dações, incorporações, permutas ou reintegrações. Entre as principais recomendações para a descrição arquivística consistem no facto de ser um elemento obrigatório na descrição de nível de fundo, sendo facultativo para os níveis inferiores. O quadro seguinte identifica as modalidades de aquisição destes bens, de acordo com o ANTT.

\begin{tabular}{|l|l|l|}
\hline Fundo & FIA & Fonte \\
\hline ALF & $\begin{array}{l}\text { Incorporação ao abrigo de Portaria do } \\
\text { Ministério do Reino. }\end{array}$ & https://digitarq.arquivos.pt/details?id=3886618 \\
\hline CFMPS & $\begin{array}{l}\text { Incorporação ao abrigo de Portaria do } \\
\text { Ministério do Reino. }\end{array}$ & https://digitarq.arquivos.pt/details?id=3909719 \\
\hline CNSPRB & {$[$ Omite] } & https://digitarq.arquivos.pt/details?id=1375741 \\
\hline CNSPSC & [Omite] & https://digitarq.arquivos.pt/details?id=1375743 \\
\hline CSBCL & [Omite] & https://digitarq.arquivos.pt/details?id=1379034 \\
\hline CSCF & [Omite] & https://digitarq.arquivos.pt/details?id=1379435 \\
\hline CSFF & [Omite] & https://digitarq.arquivos.pt/details?id=1379962 \\
\hline CSSC & [Omite] & https://digitarq.arquivos.pt/details?id=1382433 \\
\hline PJRFF & $\begin{array}{l}\text { Incorporação, em 1886, ao abrigo de } \\
\text { Portaria do Ministério do Reino. }\end{array}$ & https://digitarq.arquivos.pt/details?id=4310201 \\
\hline CSF & [Omite] & https://digitarq.arquivos.pt/details?id=1379940 \\
\hline CNSEF & [Omite] & https://digitarq.arquivos.pt/details?id=1375671 \\
\hline
\end{tabular}

Quadro 1 - Listagem dos fundos custodiados pelo ANTT. Fonte: Portal Português de Arquivos/Digitarq.

Conforme se pode verificar no Quadro 1, apenas três fundos mencionam explicitamente o critério de aquisição por "incorporação" por diploma, a Portaria do Ministério do Reino de 9 de junho de 1886, enquanto os fundos conventuais não mencionam qualquer critério, não se cumprindo, assim, o requisito estipulado pelo órgão de gestão dos arquivos em termos de descrição arquivística. No entanto, as informações sobre os processos de aquisição destes bens pelo ANTT encontram-se no metadado História Custodial $e$ Arquivistica, mencionando a legislação que determinou à transferência dos fundos do Funchal para Lisboa. Por um lado, o ANTT justifica a aquisição ao abrigo do Decreto de 2 de outubro de $1862^{59}$ que determinou a transferência e incorporação "no archivo nacional os archivo os cartorios de todas as igrejas e corporações religiosas". As consequências supervenientes deste diploma conduziram ao deslocamento de vastos conjuntos documentais de diversas regiões do país para Lisboa, com consequências

\footnotetext{
${ }^{57}$ Conselho Internacional de Arquivos, Op. cit., p. 28.

${ }^{58}$ Direcção-Geral de Arquivos, Op. cit., p. 78.

${ }^{59}$ Portugal Ministério dos Negócios Eclesiásticos e de Justiça, "Decreto de 2 de Outubro de 1862", Diário de Lisboa n. ${ }^{\circ}$ 238, Livro 1862, 1862 (versão eletrónica, consultada a 4 de janeiro de 2019, em $\mathrm{http}$ //legislacaoregia.parlamento.pt/V/1/37/92/p330).
} 
negativas ${ }^{60}$. Para além deste diploma, os IAI invocam a Portaria do Ministério do Reino de 9 de junho de 1886 nos dez fundos custodiados pelo ANTT. Apesar de o IAI referente ao fundo CNSEF omitir as bases legais que determinaram a sua transferência para o ANTT, tal informação constava em IAI anteriores. É surpreendente notar que a Portaria do Ministério do Reino de 9 de junho de 1886 não se encontra identificada nos índices do Diário de Governo de 1886. Já em 1921, os autores do Elucidário Madeirense denominavam este diploma como "portaria surda" 61 . Por que motivo o ANTT invoca nos seus IAI um diploma que não existe?

No caso do ADF, os conjuntos documentais remanescentes encontrados na Repartição de Finanças do Funchal foram incorporados em 1937 nos termos da alínea e) do número 1 do art. $^{\circ} 26 .^{\circ}$ do Decreto de 19952 , de 27 de junho. Contudo, o ABM não disponibiliza um IAI atualizado dentro das regras e convenções para a descrição arquivística, conforme já expusemos.

Em síntese, as referências sobre FIA nos IAI do ANTT não cumprem na totalidade com as normas de descrição arquivística, omitindo, por um lado, a obrigatoriedade de inclusão de informação referente ao procedimento de aquisição dos bens arquivísticos e, por outro, à menção a um diploma cujo teor se desconhece e que não é esclarecido pela instituição custodiante.

\section{Conclusões}

Nesta comunicação, fizemos uma breve incursão sobre os problemas relacionados com os "arquivos deslocados" do ponto de vista do quadro teórico e conceptual. É possível percecionar que o acesso aos IAI e fundamental para a identificação de arquivos que se encontram nesta condição ${ }^{62}$. As garantias literárias concebidas para a normalização da descrição arquivística visam assegurar a produção estruturada de IAI. Desta forma, analisámos os IAI produzidos pelo ANTT em torno dos fundos reivindicados pela Resolução da Assembleia Legislativa da Madeira n. ${ }^{\circ}$ 3/2017/M, de 12 de janeiro. Podemos tecer algumas conclusões com base nos dados atrás analisados:

- os fundos reivindicados pela Resolução já mencionada não se basearam em IAI mais atualizados;

- o ANTT já disponibiliza em ambiente web os fundos madeirenses integralmente digitalizados e acessíveis no seu portal institucional, exceto o ABM, inviabilizando uma possível reunificação digital;

- o ANTT tem realizado intervenções sucessivas na representação dos fundos reivindicados pela RAM em termos de reorganização, descrição e digitalização, apesar de as recomendações para a descrição arquivística normalizada não terem sido acatadas.

De facto, não se pode deixar de reconhecer que o ANTT continua a proporcionar o acesso à informação aos fundos reivindicados não só através dos IAI eletrónicos mas também a disponibilizar os objetos digitais das unidades descritas. Pudemos verificar que os dados

\footnotetext{
60 Paulo J. S. Barata, “As Livrarias Dos Mosteiros e Conventos Femininos Portugueses Após a Sua Extinção: Uma Aproximação a Uma História Por Fazer”, Lusitania Sacra, vol. 24, 2016, p. 125-152 (versão eletrónica, consultada a 4 de janeiro de 2019, em http://portal.cehr.ft.lisboa.ucp.pt/LusitaniaSacra/index.php/journal/article/view/209); Fernanda Ribeiro, Para o Estudo Do Paradigma Patrimonista e Custodial: A Inspecção Das Bibliotecas e Arquivos E o Contributo de António Ferrão (1887-1965), Porto: Afrontamento, 2008.

${ }^{61}$ Fernando Augusto da Silva e Carlos Azevedo de Meneses, Elucidario madeirense, vol. 1, Funchal, Tipografia Esperança, 1921.

62 Samantha R. Winn, "Ethics of Access in Displaced Archives", Provenance, Journal of the Society of Georgia Archivists, vol. 33, n. ${ }^{\circ}$ 1, 2015, p. 6-13 (versão eletrónica, consultada a 4 de janeiro de 2019, em https://digitalcommons.kennesaw.edu/provenance/vol33/iss1/5).
} 
disponibilizados pelo ANTT podem ser modificados de acordo com as conveniências da entidade custodiante. Além disto, os IAI do ANTT e do ABM não tiveram o propósito de disponibilizar uma representação inteletual e reunificada dos conjuntos documentais dispersos entre estas entidades. Deveríamos questionar se o recurso a tecnologias avançadas para os arquivos (como a digitalização) por parte da entidade custodiante não constitui uma abordagem que limita a hipótese de reunificação física sugerida pela Resolução da Assembleia Legislativa da Madeira n. ${ }^{\circ}$ 3/2017/M, de 12 de janeiro? Em que medida a disponibilização de IAI que não são construídos com a comunidade reivindicante um mecanismo intencional de limitar o acesso ao património cultural? Neste sentido, estas questões podem vir a ser desenvolvidas no âmbito teórico da crítica póscolonial, de como o uso das novas tecnologias de informação constituir uma forma de relação de poder entre entidades custodiantes e de manipulação da memória das comunidades desapossadas ${ }^{63}$.

Em última análise, os IAI podem esclarecer ou obscurecer a proveniência de conjuntos documentais que se encontram na condição de deslocados. As garantias literárias para a descrição arquivística não asseguram claramente a identificação inequívoca de bens arquivísticos que se encontram nessa condição. A este respeito, o Código de Ética do Conselho Internacional de Arquivos recomenda que os "arquivistas devem cooperar na repatriação de arquivos deslocados" ${ }^{\text {64 }}$. Contudo, há limitações, de ordem política e institucional, que coartam a atividade dos arquivistas na resolução dos "arquivos deslocados".

\footnotetext{
${ }^{63}$ James Lowry, "Radical Empathy, the Imaginary and Affect in (Post)Colonial Records: How to Break out of International Stalemates on Displaced Archives", Archival Science, 2019, 1-19 (versão eletrónica, consultada a 4 de janeiro de 2019, em https://doi.org/10.1007/s10502-019-09305-z).

${ }^{64}$ International Council on Archives, ICA Code of Ethics, General Assembly in Its XIIIth Session in Beijing (China) on 6 September 1996, 1996 (versão eletrónica, consultada a 4 de janeiro de 2019, em https://www.ica.org/en/ica-code-ethics).
} 